\title{
An animal model using Eimeria live vaccine and to study coccidiosis protozoa pathogenesis
}

\author{
Hyun-A Lee ${ }^{1,2}$, Sunhwa Hong ${ }^{1,2}$, Ohmok Choe ${ }^{1}$, Okjin Kim ${ }^{1,3, *}$ \\ ${ }^{1}$ Center for Animal Resources Development, and \\ ${ }^{2}$ Institute of Animal Experiment \& Efficacy Evaluation, and \\ ${ }^{3}$ Institute of Biotechnology, Wonkwang University, Iksan 570-749, Korea \\ (Received: June 03, 2011; Revised: September 22, 2011; Accepted: Seotember 22, 2011)
}

\begin{abstract}
Cell culture systems for the protozoan Eimeria are not yet available. The present study was conducted to develop an animal model system by inoculating animals with a live Eimeria vaccine. This study was conducted on 3-day-old chickens $(\mathrm{n}=20)$ pretreated with cyclophosphamide. The chickens were divided into 2 groups: the control group $(\mathrm{n}=10)$ and the inoculated group that received the live Eimeria vaccine $(\mathrm{n}=10)$. During the study period, we compared the clinical signs, changes in body weight, and number of oocysts shed in the feces of the control and inoculated group. This study showed that oocyst shedding was significantly higher in the chickens inoculated with live Eimeria oocysts than in the control chickens. Moreover, body weight gain was lesser in the animals in the inoculated group than in the control animals. Fecal oocyst shedding was observed in the inoculated animals. On the basis of these findings, we suggest that live Eimeria vaccination with cyclophosphamide pretreatment may be used to obtain an effective animal model for studying protozoan infections. This animal study model may eliminate the need for a tedious continuous animal inoculation process every 6 months because the live coccidiosis vaccine contains live oocysts.
\end{abstract}

Keywords : animal model, coccidiosis, cyclophosphamide, Eimeria, Protozoa

Eimeria infection causes extensive destruction of the intestinal epithelium, resulting in a reduction in feeding efficiency and body weight gain and a temporary reduction in egg production $[5,10]$. Although coccidiosis can be mainly controlled by using chemotherapeutic agents, novel treatment approaches are urgently needed because of the increasing emergence of drug-resistant parasitic strains in commercial poultry production settings $[1,2,17]$. The genus Eimeria includes obligate intracellular parasites, which are challenging to study. In vitro culture has become a useful study tool because it enables us to understand the aspects of the organisms' life cycle that could not otherwise be studied [9]. Thus far, no efficient in vitro model is available for propagating all the life cycle stages of the protozoan Eimeria. For the efficient storage of sporulated Eimeria oocysts they must be maintained by infecting chickens every 6 months as described; however, this maintenance is tedious and time-consuming [16]. A cell culture system for Eimeria species is not yet available. The present study was conducted to develop an animal model system by inoculating animals with a live Eimeria vaccine.

This study was conducted on 3-day-old chickens ( $\mathrm{n}=$ 20) at the animal facility of the Center for Animal Resources Development, Wonkwang University, Korea. The animals were acclimatized and kept in an animal facility with regulated temperature $\left(28 \pm 2^{\circ} \mathrm{C}\right)$, humidity $(50 \pm 5 \%)$, and light/dark cycle $(12 / 12 \mathrm{~h})$. The animals were fed commercial post-broiler diet without antibiotics and coccidiostats (Hanil Feed, Korea) and tap water $a d$ libitum. The chickens were kept in wire-floored grower cages during the study period. All animals were pretreated with cyclophosphamide (Sigma Chemical, USA). The cyclophosphamide (cyclophosphamide 2H-1.3, 2-oxazaphosphorin-2-amine)-pretreated chickens were injected a solution containing $3 \mathrm{mg} / \mathrm{mL}$ cyclophosphamide subcutaneously in the abdominal region daily for 4 consecutive days. Thereafter, the chickens were divided into 2

*Corresponding author

Tel: +82-63-850-6668, Fax: +82-63-850-7308

E-mail: kimoj@wku.ac.kr 
groups: the control group $(\mathrm{n}=10)$ and the inoculated group that received the live Eimeria vaccine $(\mathrm{n}=10)$. Chickens in the inoculated group were orally inoculated with the live Eimeria vaccine, Coccivac-D (Intervet/ Schering-Plough Animal Health, USA), which contains 16,000 live oocysts of Eimeria (E.) acervulina, E. mivati, E. maxima, E. tenella, E. necatrix, E. praecox, E. brunette, and $E$. hagani. Chickens in the inoculated group $(\mathrm{n}=10)$ were orally inoculated by gavage by using a 24 -gauge stainless-steel animal-feeding tube (Popper \& Sons, USA), which is used for feeding mice, attached to a 3 $\mathrm{mL}$ syringe (Fig. 1). The oral infectious dose of this vaccine has been found to be approximately $10^{4}$ oocysts of Eimeria species in $1 \mathrm{~mL}$ of saline. The control chickens $(n=10)$ received saline through the same route.

During the study period, the animals were checked twice daily for morbidity and mortality. Further, we compared the clinical signs, changes in body weight, number of oocysts shed in the feces of the control and inoculated groups. Fecal samples were collected from 6 to 10 days post-infection, and the number of oocysts was determined using a McMaster counting chamber. Total

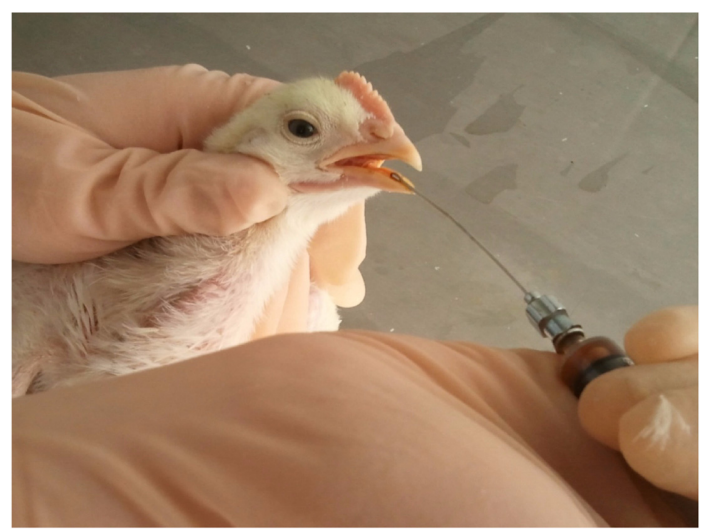

Fig. 1. The chickens were orally inoculated by gavage by using a 24-gauge stainless-steel animal-feeding tube, which is used for feeding mice, attached to a $3-\mathrm{mL}$ syringe. number of oocysts was calculated using the following formula: [total number of oocysts $=$ oocyst $\operatorname{count} \times$ dilution factor $\times$ (fecal sample volume/counting chamber volume)/number of birds per cage]. Body weights were individually measured for 2 weeks before infection and for 10 days post-infection. Differences in mean oocyst production and mean weight gain between the 4 groups were tested by using one-way analysis of variance (ANOVA; GraphPad InStat; GraphPad Software, USA) and considered significant at $p<0.05$. All studies were performed in accordance with the Guide for Animal Experimentation by Wonkwang University and were approved by the Institutional Animal Care and Use Committee of Wonkwang University (Approval No. WKU11-007). We made all efforts to minimize the pain or discomfort of the animals used in the study.

As shown in Table 1, oocyst shedding was significantly higher in the inoculated chickens than in the control chickens $(p<0.05)$. The number of fecal oocysts shed was highest on day 7 post-inoculation (Table 1). Moreover, body weight gain was lesser in the animals in the inoculated group than in the animals in the control group (Table 2). Fecal oocyst shedding was observed in the inoculated animals (Fig. 2).

The genus Eimeria includes intracellular coccidian (phylum Apicomplexa) parasites that affect the intestinal epithelial cells in chickens. The organisms are pathogenic in the asexual stages of the life cycle, and infection by these organisms clinically manifest as coccidiosis. This parasite is considered to cause the most important poultry infection worldwide [4]. We evaluated the host's susceptibility to avian coccidiosis by enumerating fecal oocysts and determining body weight changes after challenge infection with live coccidial parasites [2, 11]. Thus far, no efficient in vitro model is available for propagating all the life cycle stages of Eimeria. For the experiment, Eimeria oocysts must be maintained by subsequent inoculation in animals every 6 months, and the maintenance is tedious and time-consuming [16]. In

Table 1. Number of oocysts shed in the feces of studied chickens

\begin{tabular}{cccccc}
\hline \hline \multirow{2}{*}{ Group } & \multicolumn{5}{c}{ Oocyst number $\left(\times 10^{6}\right) /$ Days post infection } \\
\cline { 2 - 6 } & 6 & 7 & 8 & 9 & 10 \\
\hline Control & $0 \pm 0$ & $0 \pm 0$ & $0 \pm 0$ & $0 \pm 0$ & $0 \pm 0$ \\
Inoculated $^{a}$ & $12 \pm 2.71^{*}$ & $58.2 \pm 2.94^{*}$ & $22.8 \pm 2.15^{*}$ & $6.5 \pm 1.78^{*}$ & $1.3 \pm 0.67^{*}$ \\
\hline
\end{tabular}

${ }^{a}$ Chickens inoculated with live Eimeria oocysts in vaccine.

${ }^{*}$ Significantly different from control chickens $(p<0.05)$. 
Table 2. Changes in body weight of studied chickens

\begin{tabular}{cccccc}
\hline \hline \multirow{2}{*}{ Group } & \multicolumn{5}{c}{ Body weight $(\mathrm{g}) /$ Days post infection } \\
\cline { 2 - 6 } & 1 & 3 & 5 & 7 & 10 \\
\hline Control & $117.1 \pm 2.88$ & $135.3 \pm 3.40$ & $169.4 \pm 1.96$ & $215.5 \pm 2.42$ & $248.7 \pm 3.20$ \\
Inoculated $^{\mathrm{a}}$ & $114.3 \pm 1.83$ & $12.5 \pm 2.64^{*}$ & $149.8 \pm 3.01^{*}$ & $176.5 \pm 3.03^{*}$ & $216.5 \pm 3.63^{*}$ \\
\hline
\end{tabular}

${ }^{a}$ Chickens inoculated with live Eimeria oocysts in vaccine.

${ }^{*}$ Significantly different from control chickens $(p<0.05)$.
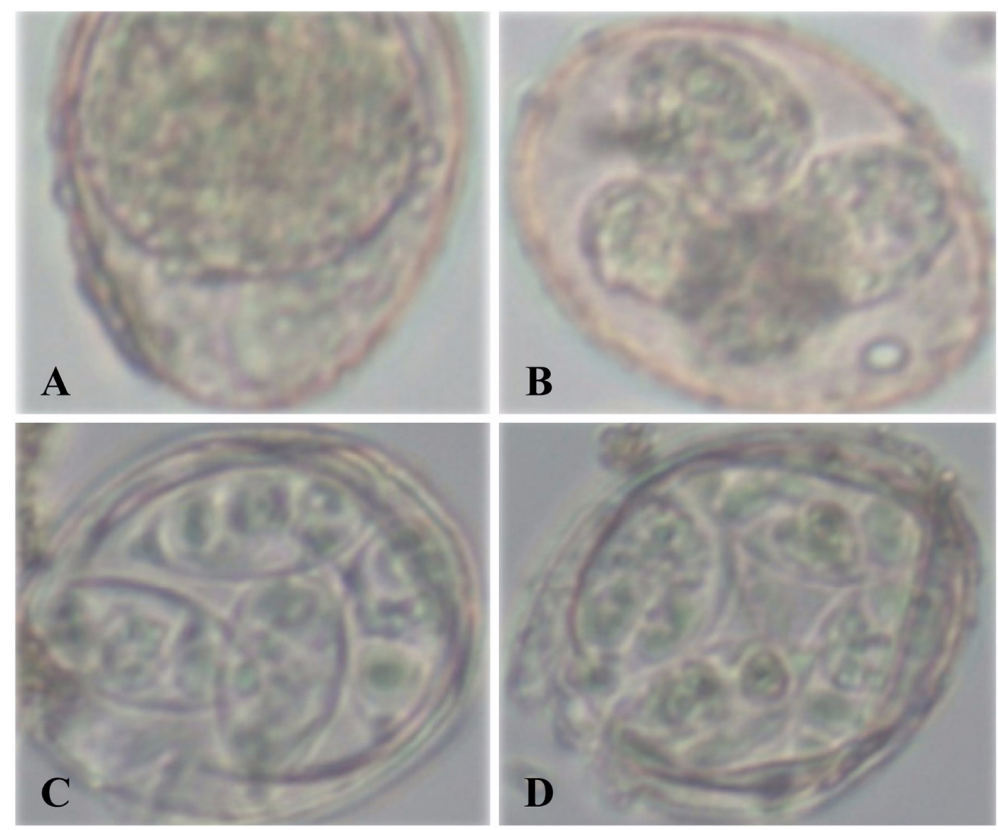

Fig. 2. Fecal oocyst shedding was observed in Eimeria-inoculated chickens.

this study, we aimed to develop an animal model system by inoculating animals with a live Eimeria vaccine that contains live Eimeria oocysts. Various immune system components such as functional activity of T- and/or Blymphocytes, humoral immune responses, and phagocytes or other cells of the immune system may be adversely affected in the immunosuppressed birds, resulting in suboptimal immune responses to vaccination or increased susceptibility to infections in the host birds $[3,8,14$, 15]. Cyclophosphamide induces humoral immunosuppression by selectively damaging the B-lymphocytes of birds and rats $[3,6,12,13]$. In this study, all animals were immunosuppressed using cyclophosphamide pretreatment. Thereafter, these immunosuppressed chickens were inoculated with live Eimeria oocysts. Fecal oocyst shedding and body weight loss were the most reliable disease parameters and were used to measure the effects of coccidiosis [2, 7]. In this study, significantly greater oocyst shedding was observed in the chickens inoculated with live Eimeria oocysts than in the control chickens. The body weights of the control and inoculated group were statistically significant.

On the basis of these results, we suggest that live Eimeria vaccine with cyclophosphamide pretreatment may be used to obtain an effective animal model for studying protozoan infections in animals. This animal study model may eliminate the need for a tedious and continuous animal inoculation process every 6 months because live coccidiosis vaccine contains live oocysts.

\section{Acknowledgments}

This study was supported by the Basic Science Research Program of the National Research Foundation 
of Korea (NRF) and funded by the Ministry of Education and Science and Technology (2010-0021940).

\section{References}

1. Allen PC, Fetterer RH. Recent advances in biology and immunobiology of Eimeria species and in diagnosis and control of infection with these coccidian parasites of poultry. Clin Microbiol Rev 2002, 15, 5865.

2. Chapman HD, Matsler PL, Muthavarapu VK, Chapman ME. Acquisition of immunity to Eimeria maxima in newly hatched chickens given 100 oocysts. Avian Dis 2005, 49, 426-429.

3. Chaudhry KM. Immunomodulatory effects of coccidiostats in broiler chicks. Master thesis. University of Agriculture, Faisalabad, Pakistan, 1991.

4. Cox FEG. Control of coccidiosis: lessons from other sporozoa. Int J Parasitol 1998, 28, 165-179.

5. Dalloul RA, Lillehoj HS. Recent advances in immunomodulation and vaccination strategies against coccidiosis. Avian Dis 2005, 49, 1-8.

6. Kim Y, Brown TP, Pantin-Jackwood MJ. The effects of cyclophosphamide treatment on the pathogenesis of subgroup $\mathbf{J}$ avian leukosis virus (ALV-J) infection in broiler chickens with Marek's disease virus exposure. J Vet Sci 2004, 5, 49-58.

7. Lillehoj HS, Trout JM. Avian gut-associated lymphoid tissues and intestinal immune responses to Eimeria parasites. Clin Microbiol Rev 1996, 9, 349-360.

8. Markowski-Grimsrud CJ, Schat KA. Infection with chicken anaemia virus impairs the generation of pathogen-specific cytotoxic T lymphocytes. Immunology 2003, 109, 283-294.
9. McDougald LR. The growth of avian Eimeria in vitro. In: Long PL, Boorman KN, Freeman BM (eds.). Avian Coccidiosis. pp. 185-223, British Poultry Science, Edinburgh, 1978.

10. Min W, Dalloul RA, Lillehoj HS. Application of biotechnological tools for coccidia vaccine development. J Vet Sci 2004, 5, 279-288.

11. Min W, Lillehoj HS, Burnside J, Weining KC, Staeheli P, Zhu JJ. Adjuvant effects of $I L-1 \beta, I L-2$, $I L-8, I L-15, I F N-\alpha, I F N-\gamma, T G F-4$ and lymphotactin on DNA vaccination against Eimeria acervulina. Vaccine 2001, 20, 267-274.

12. Misra RR, Bloom SE. Roles of dosage, pharmacokinetics, and cellular sensitivity to damage in the selective toxicity of cyclophosphamide towards B and T cells in development. Toxicology 1991, 66, 239-256.

13. Muruganandan S, Lal J, Gupta PK. Immunotherapeutic effects of mangiferin mediated by the inhibition of oxidative stress to activated lymphocytes, neutrophils and macrophages. Toxicology 2005, 215, 57-68.

14. Naeem K, Niazi T, Malik SA, Cheema АH. Immunosuppressive potential and pathogenicity of an avian adenovirus isolate involved in hydropericardium syndrome in broilers. Avian Dis 1995, 39, 723-728.

15. Sharma JM, Kim IJ, Rautenschlein S, Yeh HY. Infectious bursal disease virus of chickens: pathogenesis and immunosuppression. Dev Comp Immunol 2000, 24, 223-235.

16. Shirley MW. Eimeria species and strains of chicken. In: Eckert J, Braun R, Shirley MW, Coudert P (eds.). Guidelines on Techniques in Coccidiosis Research. pp. 1-25, European Commission, Luxemburg, 1995.

17. Williams RB. Fifty years of anticoccidial vaccines for poultry (1952-2002). Avian Dis 2002, 46, 775-802. 